Research Paper

\title{
The Lymphocyte-Monocyte Ratio Predicts Patient Sur- vival and Aggressiveness of Endometrial Cancer
}

\author{
Wan Kyu Eo', Sanghoon Kwon², Suk Bong Koh³, Min Jeong Kim, Yong Il Ji5, Ji Young Lee', Dong Soo \\ Suh $^{7}$, Ki Hyung Kim ${ }^{7 *}$, Heung Yeol Kim ${ }^{8^{*}} \bowtie$ \\ 1. Department of Internal Medicine, College of Medicine, Kyung Hee University, Seoul, Korea \\ 2. Department of Obstetrics and Gynecology, Keimyung University, School of Medicine, Daegu, Korea \\ 3. Department of Obstetrics and Gynecology, Catholic University of Daegu, School of Medicine, Daegu, Korea \\ 4. Department of Obstetrics and Gynecology, The Catholic University of Korea, Seoul, Korea \\ 5. Department of Obstetrics and Gynecology, College of Medicine, Inje University, Busan, Korea \\ 6. Department of Obstetrics and Gynecology, Research Institute of Medical Science, Konkuk University School of Medicine, Seoul, Korea \\ 7. Department of Obstetrics and Gynecology, Pusan National University School of Medicine; Biomedical Research Institute, Pusan National University Hos- \\ pital, Busan, Korea \\ 8. Department of Obstetrics and Gynecology, College of Medicine, Kosin University, Busan, Korea \\ * Ki Hyung Kim and Heung Yeol Kim equally contributed to this work.
}

$\triangle$ Corresponding authors: Ki Hyung Kim, M.D., Ph.D., Department of Obstetrics and Gynecology, Pusan National University School of Medicine; Biomedical Research Institute, Pusan National University Hospital, Busan 602-739, Korea; Phone: +82-51-990-6226; Fax: +82-51-990-3300; e-mail: kihyungkimpnu@gmail.com. Heung Yeol Kim, M.D., Ph.D., Department of Obstetrics and Gynecology, Kosin University College of Medicine, 34, Amnam-dong, Seo-gu, Busan 602-702, Korea; Phone: +82-51-990-6211; Fax: +82-51-241-5458; E-mail: hykyale@yahoo.com

(C) Ivyspring International Publisher. Reproduction is permitted for personal, noncommercial use, provided that the article is in whole, unmodified, and properly cited. See http://ivyspring.com/terms for terms and conditions.

Received: 2015.10.23; Accepted: 2016.01.22; Published: 2016.02.20

\begin{abstract}
Objective: We assessed the prognostic implications of preoperative lymphocyte-monocyte ratio (LMR) in patients with endometrial cancer (EC).

Methods: We retrospectively examined the LMR as a prognostic variable in a cohort of 255 patients with EC who underwent surgical resection. Patients were categorized into two groups according to the LMR (LMR-low and LMR-high) using cutoff points determined by receiving operator characteristic (ROC) curve analysis. The primary objective was to correlate the LMR to clinicopathological factors; the secondary objective was to determine the survival significance of the LMR in patients with EC.

Results: Using data from the entire cohort, the most discriminative LMR cutoff value selected on the ROC curve was 3.28 for both disease-free survival (DFS) and overall survival (OS). The LMR-low and LMR-high groups included 33 (12.9\%) and 222 patients (87.1\%), respectively. The 5 -year DFS rates in the LMR-low and LMR-high groups were 64.5 and $93.9 \%(P<0.0001)$, respectively, and the 5 -year OS rates in the two groups were 76.7 and $96.5 \%(P<0.0001)$, respectively. On multivariate analysis, we identified histologic grade, International Federation of Gynecology and Obstetrics (FIGO) stage, and LMR levels as the strongest prognostic factors affecting DFS $(P=0.0037, P<0.0001$, and $P<0.0001$, respectively), and FIGO stage and the LMR as the strongest prognostic factors predicting OS $(P<0.0001$ and $P<0.0001$, respectively).

Conclusion: The LMR is an independent prognostic factor for both DFS and OS after surgical resection, and it provides additional prognostic value beyond standard clinicopathological parameters.
\end{abstract}

Key words: Monocytes, Lymphocytes, Endometrial Cancer

\section{Introduction}

Endometrial cancer (EC) is the most common gynecologic malignancy in Europe and North Amer- ica, and the incidence of this disease and the associated mortality have increased over the past decade 
[1]. The majority of ECs present as low-grade tumors that tend to show limited spread to the surface of the endometrium. In addition, most patients with EC present with symptoms of unusual vaginal bleeding, and this enables early diagnosis of malignancy [1]. As the majority of ECs are low-grade tumors detected at an early stage, surgery followed by tailored adjuvant therapy based on the patient's clinicopathological risk profile is the standard initial treatment for this disease. Owing to the favorable tumor characteristics of EC, a long period of remission with a 5-year survival rate of greater than $80 \%$ is observed [2], and a cure for the disease is possible in the majority of patients.

However, despite a multidisciplinary treatment approach involving surgery, chemotherapy, and radiotherapy, a significant number of patients suffer from recurrent disease; the risk of recurrence for EC patients is $10-20 \%$ for International Federation of Gynecology and Obstetrics (FIGO) stage I-II disease and $50-70 \%$ for stage III-IV disease [3]. Therefore, novel approaches to identify tumors that are likely to recur may allow for optimization of treatment in these patients, along with improved survival. Several clinicopathological models have been proposed to identify patients at risk of relapse of EC and subsequent death, and these strategies have the ultimate objective of identifying individuals who would derive the greatest benefit from postoperative therapeutic intervention.

In patients with EC, prognosis is guided by analysis of various cancer-related risk factors: advanced FIGO stage, myometrial invasion [4-6], cervical stromal invasion (CSI) [7], extrauterine disease [6], positive peritoneal cytology [7], lymphovascular space invasion (LVSI) [8], positive pelvic nodes, positive para-aortic nodes [9], grade 3 histology [9], cancer antigen 125 (CA-125) level [10], and completeness of surgical resection. However, it is clear that the ability of these conventional risk factors to predict recurrence and estimate survival is insufficient. Clinical outcomes of patients with EC are influenced not only by cancer-related risk factors, but also by host-related risk factors including white blood cells (WBCs) [11], monocyte counts [12], hemoglobin concentration [13], platelet counts [14], the neutrophil-lymphocyte ratio (NLR) [15-17], and the platelet-lymphocyte ratio (PLR) $[15,17]$.

Recently, the lymphocyte-monocyte ratio (LMR; calculated as the proportional ratio of the absolute count of lymphocytes over the absolute count of monocytes) has been suggested to be associated with survival in patients with malignant lymphomas [18-20] as well as many solid tumors, such as head and neck [21-23], breast [24], lung [25-27], gastrointestinal tract [28-35], and genitourinary system [36, 37] cancers. However, as far as we know, the prognostic value of the LMR in patients with EC has not been reported. The primary objective of the analysis was to assess the correlation between LMR and clinicopathological factors. The secondary objective of the analysis was to determine the survival significance of the LMR in patients with EC.

\section{Methods}

This study included 255 newly diagnosed EC patients with histologically confirmed disease who were treated with hysterectomy-based comprehensive surgical staging at university hospitals between January 2005 and December 2014. Excluded cases included those without laboratory results at the time of cancer diagnosis. Patients were also excluded if the blood test including complete blood count (CBC) is not performed within 2 weeks before surgery. In addition, those who had been treated with radiation therapy or neoadjuvant chemotherapy before surgery were also excluded from this study. Patients with prior malignancies within the previous 5 years or concurrent second malignancies were also excluded. Finally, patients were ineligible if they had evidence of active infection, had used recombinant human granulocyte-macrophage colony-stimulating factor or recombinant human granulocyte colony-stimulating factor in the treatment of neutropenia, had a concomitant autoimmune disease, or had been treated with immunosuppressive therapy that may affect WBC count. The Institutional Review Board approved the retrospective review of these records, and this study was performed in accordance with local (Korean regulations) and international (the Declaration of Helsinki) ethical standards.

Clinicopathological variables such as age, histologic type, histologic grade, FIGO stage, lymph node (LN) metastasis, CSI, and presence of LVSI were obtained retrospectively from patient medical records. Staging surgery consisting of total hysterectomy, bilateral salpingo-oophorectomy, peritoneal washing, and pelvic LN dissection with or without para-aortic LN dissection was performed as the primary treatment for EC [38]. Classification of histologic type was reviewed for consistency by a single pathologist. Subtypes included endometrioid, serous, mucinous, clear cell, mixed cell, and other tumors, and histological diagnosis was determined based on World Health Organization (WHO) histological classification guidelines [39]. Histologic grade was based on the FIGO system, and cancer stage was reclassified based on the 2009 FIGO staging system [38]. Adjuvant treatment was administered depending on risk factors (FIGO stage, histologic type, and histologic grade), patient preference, and physician discretion [40]. 
Laboratory results for CBCs included WBC count; absolute neutrophil count (ANC), absolute lymphocyte count (ALC), and absolute monocyte count (AMC); hemoglobin levels; mean corpuscular volume (MCV); and platelet counts. In addition, biochemical results for CA-125 and serum albumin levels were abstracted. Laboratory measurements were performed prior to surgery as part of the routine workup. If more than one preoperative $\mathrm{CBC}$ results were available, the result from the date closest to the surgical procedure was chosen for analysis [41].

As the optimized cutoff values for the NLR $[15-17,42]$ and PLR $[15,17]$ were variable in previous studies, and no prior study examined the influence of the LMR on survival in patients with EC, we used data from the entire cohort to determine best cutoff points for predicting disease-free (DFS) and overall survival (OS) based on receiver operating characteristic (ROC) curve analysis. We determined that the best LMR cutoff value for both DFS and OS was 3.28. Then the patients were grouped based on the results of ROC curve analysis into an LMR-low group (LMR $\leq$ 3.28) and an LMR-high group (LMR > 3.28). Differences in tumor- and host-related risk factors including age, histologic type, histologic grade, FIGO stage, LN metastasis, CSI, LVSI, and serum CA-125 and serum albumin levels between the LMR-low and LMR-high groups were analyzed. Independent-samples $t$-tests were used to assess continuous variables, whereas independent-samples chi-squared tests were used to assess categorical variables.

We also evaluated the impact of the difference in the LMR between groups on both DFS and OS. DFS was defined as the time interval between hysterectomy-based surgical staging and the date of first recurrence or the date of last follow-up if there was no recurrence. OS was defined as the time interval between the date of hysterectomy-based surgical staging and the date of death due to any cause or last follow-up. Patients who did not experience cancer recurrence or death were censored at the time of last known contact date. The Kaplan-Meier method was used for descriptive analysis of survival curves; survival curves were compared using log-rank tests. We used the univariate Cox proportional hazards model for identifying the contribution of the following variables: age, histologic type, histologic grade, FIGO stage, LVSI, serum CA-125 and serum albumin levels, WBC count, ANC, ALC, AMC, hemoglobin level, MCV, platelet count, NLR, PLR, and LMR. The multivariate Cox proportional hazards models were used to determine adjusted hazard ratios for survival. Variables with $P$-values $<0.1$ were selected for the multivariate analysis. All presented $P$-values are two-sided, and statistical significance was declared at
$P<0.05$. Data were analyzed using Statistical Package for the Social Science (SPSS) statistical software, version 18.0 (SPSS Inc., Chicago, IL, USA).

\section{Results}

The baseline characteristics of the patients are displayed in Table 1. Endometrioid adenocarcinoma was the most common histological subtype (91.8\%), and histologic grade 1 was the most frequent grade $(50.0 \%)$ in our cohort. In total, $190(74.5 \%), 25$ (9.8\%), $35(13.7 \%)$, and $5(2.0 \%)$ patients had stage I, II, III, and IV disease, respectively. LN involvement and CSI were observed in $31(12.2 \%)$ and $45(17.6 \%)$ patients, respectively. Forty-seven $(18.4 \%)$ patients were found to have LVSI. The median serum level of CA-125 was 19 units $/ \mathrm{mL}$, and the median serum albumin level was $4.4 \mathrm{~g} / \mathrm{dL}$.

Table 1. Clinicopathological characteristics of 255 patients with endometrial cancer

\begin{tabular}{|c|c|}
\hline Variable & $n(\%)$ \\
\hline Age (years), median (range) & $44(28-82)$ \\
\hline \multicolumn{2}{|l|}{ Histology } \\
\hline Endometrioid & $234(91.8)$ \\
\hline Serous & $7(2.7)$ \\
\hline Mixed & $7(2.7)$ \\
\hline Clear cell & $3(1.2)$ \\
\hline Undifferentiated & $2(0.8)$ \\
\hline Mucinous & $1(0.4)$ \\
\hline Squamous & $1(0.4)$ \\
\hline \multicolumn{2}{|l|}{ Histologic grade } \\
\hline G1 & $127(50.0)$ \\
\hline G2 & $88(34.6)$ \\
\hline G3 & $39(15.4)$ \\
\hline \multicolumn{2}{|l|}{ FIGO Stage } \\
\hline I-II & $215(84.3)$ \\
\hline III-IV & $40(15.7)$ \\
\hline \multicolumn{2}{|l|}{ LN metastasis } \\
\hline Absent & $224(87.8)$ \\
\hline Present & $31(12.2)$ \\
\hline \multicolumn{2}{|l|}{ CSI } \\
\hline Absent & $210(82.4)$ \\
\hline Present & $45(17.6)$ \\
\hline \multicolumn{2}{|l|}{ LVSI } \\
\hline Absent & $208(81.6)$ \\
\hline Present & $47(18.4)$ \\
\hline CA-125 (unit/mL), median (range) & $19.0(5.2-1144.0)$ \\
\hline Albumin (g/dL), median (range) & $4.4(2.2-5.3)$ \\
\hline WBC (per $\mu \mathrm{L})$, median (range) & $6700(3080-25900)$ \\
\hline ANC (per $\mu \mathrm{L})$, median (range) & $3929.3(1653.9-21833.7)$ \\
\hline ALC (per $\mu \mathrm{L})$, median (range) & $1979.3(366.6-4498.5)$ \\
\hline AMC (per $\mu \mathrm{L})$, median (range) & $357.3(72.4-2201.5)$ \\
\hline Hemoglobin $(\mathrm{g} / \mathrm{dL})$, median (range) & $12.7(6.2-15.7)$ \\
\hline MCV (fL), median (range) & $89.3(63.7-98.5)$ \\
\hline Platelet $\left(\times 10^{3} / \mu \mathrm{L}\right)$, median (range) & $264.0(73.0-571.0)$ \\
\hline
\end{tabular}


When patients were stratified according to the LMR, the LMR-low and LMR-high groups included $33(12.9 \%)$ and $222(87.1 \%)$ patients, respectively. To evaluate the relevance of the LMR, we assessed differences in the baseline characteristics of the patients according to the different LMR categories. Significant mean differences between the LMR-low and LMR-high groups were demonstrated for the following continuous variables: serum albumin levels $(P<$ $0.0001)$, WBC count $(P<0.0001)$, ANC $(P<0.0001)$, ALC $(P<0.0001)$, AMC $(P<0.0001)$, hemoglobin concentration $(P=0.0016)$, NLR $(P<0.0001)$, and PLR $(P<0.0001)$. In addition, significant differences in categorical variables included histologic type $(P=$ $0.0259)$, FIGO stage $(P=0.0133)$, and LVSI $(P=0.0180)$ (Table 2).

The median duration of follow-up was 51.3 months (range, 1.0-130.0 months). Univariate analysis for DFS identified a significant difference in several variables: age $(P=0.0085)$, histologic type $(P<0.0001)$, histologic grade $(P=0.0014)$, FIGO stage $(P<0.0001)$, LVSI $(P<0.0848)$, CA-125 levels $(P<0.0001)$, serum albumin levels $(P=0.0138), \mathrm{WBC}$ count $(P=0.0075)$, $\operatorname{ANC}(P=0.0478), \operatorname{ALC}(P=0.0019), \operatorname{AMC}(P<0.0001)$, hemoglobin concentration $(P=0.0079), \operatorname{MCV}(P=$ $0.0139)$, platelet count $(P=0.0282), \operatorname{NLR}(P=0.0032)$, $\operatorname{PLR}(P=0.0108)$, and LMR $(P<0.0001)$. Using the multivariate Cox proportional hazards model, we identified histologic grade (hazard ratio $[\mathrm{HR}]=9.57$, $95 \%$ confidence interval $[\mathrm{CI}]=2.08-44.01, P=0.0037$ ),
FIGO stage $(\mathrm{HR}=8.14,95 \% \mathrm{CI}=3.14-21.11, P<$ 0.0001 ), and LMR (HR $=0.10,95 \% \mathrm{CI}=0.03-0.22, P<$ 0.0001 ) as the strongest prognostic factors (Table 3 ).

Using univariate analysis for OS, significant differences for variables were obtained for several variables: histologic type $(P<0.0001)$, FIGO stage $(P<$ $0.0001)$, LVSI $(P<0.0001)$, CA-125 levels $(P<0.0001)$, serum albumin levels $(P=0.0460), \operatorname{ALC}(P=0.0117)$, AMC $(P=0.0042)$, hemoglobin concentration $(P<$ $0.0001)$, NLR $(P=0.0217)$, PLR $(P=0.0497)$, and LMR $(P<0.0001)$. In multivariate analyses using Cox proportional hazards model for OS, FIGO stage $(\mathrm{HR}=$ 18.67, 95\% CI $=4.08-85.50, P<0.0001)$, and LMR (HR $=0.07,95 \% \mathrm{CI}=0.02-0.24, P<0.0001)$ were identified as significant prognostic factors (Table 4).

According to Kaplan-Meier analysis, the 5-year DFS rates for patients with histologic grade 1 and grade 2-3 disease were 98.3 and $81.5 \%(P=0.0001)$, respectively, and the 5-year OS rates in these groups were 100.0 and $88.4 \%$, respectively $(P=0.0001)$. In addition, the 5-year DFS rates for patients with stage I-II and III-IV disease were 95.9 and 53.2\% $(P<$ 0.0001 ), respectively, and the 5 -year OS rates in these two patient groups were 99.4 and $64.3 \%$, respectively $(P<0.0001)$. Finally, the 5-year DFS rates in the LMR-low and LMR-high groups were 64.5 and $93.9 \%$ $(P<0.0001)$, respectively, and the 5 -year OS rates in these two groups were 76.7 and $96.5 \%$, respectively $(P$ $<0.0001$ ) (Fig. 1).

Table 2. Clinical and pathological characteristics according to the LMR in 255 patients with endometrial cancer

\begin{tabular}{|c|c|c|c|c|c|c|}
\hline \multirow[t]{2}{*}{ Variable } & & \multicolumn{2}{|r|}{ LMR-low $(\leq 3.28)$} & \multicolumn{2}{|r|}{ LMR-high (> 3.28) } & \multirow[t]{2}{*}{$P$-value } \\
\hline & & $\overline{n(\%)}$ & Mean \pm SD & $n(\%)$ & Mean \pm SD & \\
\hline Age (years) & & 33 & $56.1 \pm 11.3$ & 222 & $54.8 \pm 9.5$ & 0.5017 \\
\hline \multirow[t]{2}{*}{ Histology } & Endometrioid & 27 & & 207 & & 0.0259 \\
\hline & Non-endometrioid & 6 & & 15 & & \\
\hline \multirow[t]{2}{*}{ Histologic grade } & G1 & 16 & & 111 & & 1.0000 \\
\hline & G2-G3 & 16 & & 111 & & \\
\hline \multirow[t]{2}{*}{ FIGO stage } & I-II & 23 & & 192 & & 0.0133 \\
\hline & III-IV & 10 & & 30 & & \\
\hline \multirow[t]{2}{*}{ LN metastasis } & Absent & 26 & & 198 & & 0.0880 \\
\hline & Present & 7 & & 24 & & \\
\hline \multirow[t]{2}{*}{ CSI } & Absent & 24 & & 186 & & 0.1201 \\
\hline & Present & 9 & & 36 & & \\
\hline \multirow[t]{2}{*}{ LVSI } & Absent & 22 & & 186 & & 0.0180 \\
\hline & Present & 11 & & 36 & & \\
\hline CA-125 (unit/mL) & & 31 & $74.3 \pm 210.9$ & 217 & $39.0 \pm 73.9$ & 0.0705 \\
\hline Albumin (g/dL) & & 33 & $3.9 \pm 0.7$ & 222 & $4.4 \pm 0.4$ & $<0.0001$ \\
\hline WBC (per $\mu \mathrm{L})$ & & 33 & $9200.0 \pm 4624.2$ & 222 & $6931.7 \pm 1990.0$ & $<0.0001$ \\
\hline $\mathrm{ANC}($ per $\mu \mathrm{L})$ & & 33 & $6986.8 \pm 4326.1$ & 222 & $4228.5 \pm 1757.2$ & $<0.0001$ \\
\hline ALC (per $\mu \mathrm{L}$ ) & & 33 & $1397.5 \pm 484.3$ & 222 & $2137.7 \pm 654.8$ & $<0.0001$ \\
\hline $\mathrm{AMC}($ per $\mu \mathrm{L})$ & & 33 & $637.3 \pm 335.4$ & 222 & $364.7 \pm 132.0$ & $<0.0001$ \\
\hline Hemoglobin $(\mathrm{g} / \mathrm{dL})$ & & 33 & $11.6 \pm 2.1$ & 222 & $12.6 \pm 1.5$ & 0.0016 \\
\hline MCV (fL) & & 33 & $86.8 \pm 7.6$ & 222 & $87.9 \pm 6.4$ & 0.3507 \\
\hline Platelet $\left(\times 10^{3} / \mu \mathrm{L}\right)$ & & 33 & $267.7 \pm 92.9$ & 222 & $276.6 \pm 74.3$ & 0.5336 \\
\hline NLR & & 33 & $5.8 \pm 4.6$ & 222 & $2.2 \pm 1.8$ & $<0.0001$ \\
\hline PLR & & 33 & $205.3 \pm 76.4$ & 222 & $142.1 \pm 64.4$ & $<0.0001$ \\
\hline
\end{tabular}

$P$-values for comparison of mean differences in continuous variables were obtained by $t$-test; $P$-values for independent tests of categorical variables were obtained by chi-square test. LMR, lymphocyte monocyte ratio; SD, standard deviation; FIGO, International Federation of Gynecology and Obstetrics; LN, lymph node; CSI, cervical stromal invasion; LVSI, lymphovascular space invasion; CA-125, cancer antigen 125; WBC, white blood cell; ANC, absolute neutrophil count; ALC, absolute lymphocyte count; AMC, absolute monocyte count; MCV, mean corpuscular volume; NLR, neutrophil-lymphocyte ratio; PLR, platelet-lymphocyte ratio 
Table 3. Relationship between tumor- and host-related characteristics and disease-free survival in 255 patients with endometrial cancer

\begin{tabular}{|c|c|c|c|c|}
\hline \multirow[b]{2}{*}{ Variable } & \multicolumn{2}{|c|}{ Univariate } & \multicolumn{2}{|c|}{ Multivariate } \\
\hline & $\mathrm{HR}(95 \% \mathrm{CI})$ & $P$-value & $\mathrm{HR}(95 \% \mathrm{CI})$ & $P$-value \\
\hline Age (years) ( $\leq 56$ vs. $>56)$ & $3.59(1.39,9.29)$ & 0.0085 & & \\
\hline Histology (endometrioid vs. others) & $7.81(3.05,19.97)$ & $<0.0001$ & & \\
\hline Histologic grade (G1 vs. G2-G3) & $10.81(2.52,46.41)$ & 0.0014 & $9.57(2.08,44.01)$ & 0.0037 \\
\hline FIGO stage (I-II vs. III-IV) & $15.09(6.03,37.78)$ & $<0.0001$ & $8.14(3.14,21.11)$ & $<0.0001$ \\
\hline LVSI (absent vs. present) & $8.29(3.43,20.03)$ & $<0.0001$ & & \\
\hline CA-125 (unit/mL) ( $\leq 48.1$ vs. $>48.1)$ & $5.54(2.20,13.97)$ & $<0.0001$ & & \\
\hline Albumin (g/dL) ( $\leq 4.4$ vs. $>4.4)$ & $0.21(0.06,0.73)$ & 0.0138 & & \\
\hline WBC $($ per $\mu \mathrm{L})(\leq 5410$ vs. $>5410)$ & $5.86(0.79,43.31)$ & 0.0848 & & \\
\hline ANC $($ per $\mu \mathrm{L})(\leq 3665.1$ vs. $>3665.1)$ & $3.01(1.01,8.94)$ & 0.0478 & & \\
\hline ALC (per $\mu \mathrm{L})(\leq 1526.9$ vs. $>1526.9)$ & $0.25(0.10,0.60)$ & 0.0019 & & \\
\hline AMC $($ per $\mu \mathrm{L})(\leq 528.4$ vs. $>528.4)$ & $4.83(2.01,11.64)$ & $<0.0001$ & & \\
\hline Hemoglobin (g/dL) ( $\leq 11.7$ vs. $>11.7)$ & $0.31(0.13,0.74)$ & 0.0079 & & \\
\hline $\operatorname{MCV}(\mathrm{fL})(\leq 90$ vs. $>90)$ & $3.16(1.26,7.91)$ & 0.0139 & & \\
\hline Platelet $\left(\times 10^{3} / \mu \mathrm{L}\right)(\leq 204$ vs. $>204)$ & $0.34(0.13,0.89)$ & 0.0282 & & \\
\hline $\operatorname{NLR}(\leq 2.44$ vs. $>2.44)$ & $3.68(1.55,8.76)$ & 0.0032 & & \\
\hline PLR ( $\leq 190.78$ vs. $>190.78)$ & $3.08(1.30,7.32)$ & 0.0108 & & \\
\hline $\operatorname{LMR}(\leq 3.28$ vs. $>3.28)$ & $0.10(0.04,0.25)$ & $<0.0001$ & $0.10(0.03,0.32)$ & $<0.0001$ \\
\hline
\end{tabular}

HRs were obtained from Cox's proportional hazard model.

HR, hazard ratio; CI, confidence interval; FIGO, International Federation of Gynecology and Obstetrics; LVSI, lymphovascular space invasion;CA-125, cancer antigen 125; WBC, white blood cell; ANC, absolute neutrophil count; ALC, absolute lymphocyte count; AMC, absolute monocyte count; MCV, mean corpuscular volume; NLR, neutrophil-lymphocyte ratio; PLR, platelet-lymphocyte ratio; LMR, lymphocyte-monocyte ratio
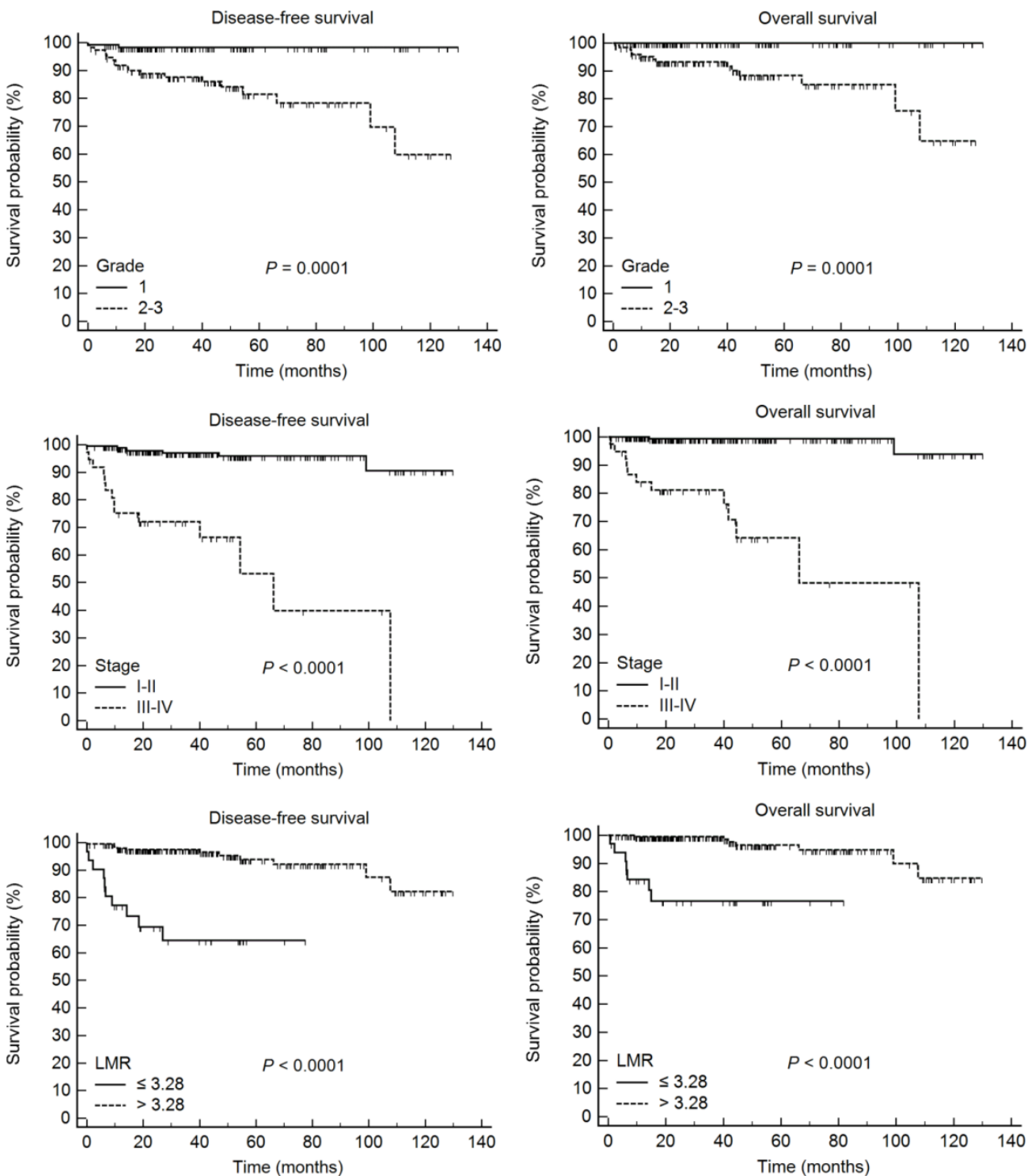

Figure 1. Disease-free survival and overall survival according to the histological grade, FIGO stage, and lymphocyte-monocyte ratio in 255 patients with endometrial cancer. 
Table 4. Relationship between tumor- and host-related characteristics and overall survival in 255 patients with endometrial cancer

\begin{tabular}{|c|c|c|c|c|}
\hline \multirow[b]{2}{*}{ Variable } & \multicolumn{2}{|c|}{ Univariate } & \multicolumn{2}{|c|}{ Multivariate } \\
\hline & HR $(95 \%$ CI) & $P$-value & HR $(95 \% \mathrm{CI})$ & $P$-value \\
\hline Age (years) ( $\leq 56$ vs. $>56)$ & $2.55(0.85,7.67)$ & 0.0960 & & \\
\hline Histology (endometrioid vs. others) & $12.868(4.13,40.02)$ & $<0.0001$ & & \\
\hline Histologic grade (G1 vs. G2-G3) & $69.88(0.94,5173.32)$ & 0.0531 & & \\
\hline FIGO stage (I-II vs. III-IV) & $45.27(10.00,204.82)$ & $<0.0001$ & $18.67(4.08,85.50)$ & $<0.0001$ \\
\hline LVSI (absent vs. present) & $16.86(4.70,60.49)$ & $<0.0001$ & & \\
\hline CA-125 (unit/mL) ( $\leq 48.1$ vs. $>48.1)$ & $6.76(2.23,20.51)$ & $<0.0001$ & & \\
\hline Albumin $(\mathrm{g} / \mathrm{dL})(\leq 4.4 \mathrm{vs} .>4.4)$ & $0.22(0.05,0.97)$ & 0.0460 & & \\
\hline WBC $($ per $\mu \mathrm{L})(\leq 5410$ vs. $>5410)$ & $3.52(0.46,27.04)$ & 0.2260 & & \\
\hline ANC $($ per $\mu \mathrm{L})(\leq 3665.1$ vs. $>3665.1)$ & $2.47(0.69,8.85)$ & 0.1663 & & \\
\hline ALC $($ per $\mu \mathrm{L})(\leq 1526.9$ vs. $>1526.9)$ & $0.26(0.09,0.74)$ & 0.0117 & & \\
\hline $\mathrm{AMC}($ per $\mu \mathrm{L})(\leq 528.4$ vs. $>528.4)$ & $4.86(1.65,13.32)$ & 0.0042 & & \\
\hline Hemoglobin (g/dL) ( $\leq 11.7$ vs. $>11.7)$ & $0.14(0.04,0.44)$ & $<0.0001$ & & \\
\hline MCV (fL) ( $\leq 90$ vs. > 90) & $3.01(0.99,9.13)$ & 0.0513 & & \\
\hline Platelet $\left(\times 10^{3} / \mu \mathrm{L}\right)(\leq 204$ vs. $>204)$ & $0.32(0.09,1.07)$ & 0.0638 & & \\
\hline $\operatorname{NLR}(\leq 2.44$ vs. $>2.44)$ & $3.47(1.20,10.05)$ & 0.0217 & & \\
\hline PLR ( $\leq 190.78$ vs. $>190.78)$ & $2.89(1.00,8.38)$ & 0.0497 & & \\
\hline LMR ( $\leq 3.28$ vs. $>3.28)$ & $0.02(0.01,0.55)$ & $<0.0001$ & $0.07(0.02,0.24)$ & $<0.0001$ \\
\hline
\end{tabular}

HRs were obtained from Cox's proportional hazard model.

HR, hazard ratio; CI, confidence interval; FIGO, International Federation of Gynecology and Obstetrics; LVSI, lymphovascular space invasion;CA-125, cancer antigen 125; WBC, white blood cell; ANC, absolute neutrophil count; ALC, absolute lymphocyte count; AMC, absolute monocyte count; NLR, neutrophil-lymphocyte ratio; PLR, platelet-lymphocyte ratio; LMR, lymphocyte-monocyte ratio

\section{Discussion}

Cancer of the endometrium is now the most frequently diagnosed gynecologic cancer in the developed world [1]. The majority of ECs present as low-grade tumors with a low risk for extrauterine spread and favorable survival outcomes. In addition, abnormal bleeding from the vagina is an early sign of EC, and thus the majority of patients are diagnosed with stage I disease [1]. Because of the favorable tumor characteristics in EC, with frequent low-grade tumors and early-stage disease at the time of presentation, long periods of remission and even cure are possible in the majority of patients. However, despite a multidisciplinary treatment approach with surgery, chemotherapy, and radiotherapy, certain patients with EC do develop disease recurrence, and cure in these cases can be quite challenging. Therefore, novel approaches for identifying tumors that are likely to recur may allow for optimization of treatment in these patients, along with improved survival.

The association between inflammation and cancer was first described by Virchow in 1863 [43], and emerging evidence has highlighted the importance of chronic inflammation in the malignant transformation, promotion, and metastasis of cancer [44, 45]. In previous clinical studies, pretreatment numbers of peripheral blood cells, including neutrophils, lymphocytes, and monocytes, have been found to be significantly associated with the progression and survival in several different kinds of cancers [24, 37, 46]. Furthermore, in recent years, several prognostic indicators derived from peripheral blood, such as the NLR, PLR, and LMR, have been widely investigated as potentially useful prognostic markers in cancers.
Despite inconsistent results from several clinical trials, these markers allegedly have significant diagnostic and prognostic value in a wide variety of cancer conditions. The NLR has been demonstrated to be a prognostic parameter for various cancer types. In EC, an elevated NLR was found to predict poor OS [15, 16] on multivariate analysis. In addition, elevated PLR was found to significantly affect the OS of women with EC on multivariate analysis [15]. In the present study, the prognostic impact of the NLR and PLR on DFS and OS was demonstrated on univariate analysis, but the significance of the associations was lost on multivariate analysis (Tables 3 and 4), as has been reported by $\mathrm{Li}$ et al. [47]. The possible reasons for the discrepant findings for the NLR and PLR may relate to the fact that optimized cutoffs were quite different between studies.

The LMR has been suggested to be associated with survival in patients with malignant lymphomas [18-20] and many solid tumors, such as head and neck [21-23], breast [24], lung [25-27], esophageal [28, 29], gastric [30,31], colorectal [32, 33], pancreatic [34, 35], bladder [36], and cervical cancers [37]. The cutoff values for the LMR were determined by ROC curve analysis in most studies, and these values ranged from 2.6 to 5.1. A low LMR was found to be associated with poor OS in previous studies $[18,19,21,23,25-29$, 32-37], and the LMR can be considered a potential surrogate biomarker in various cancers. The findings of the present study demonstrate that the LMR is a surrogate marker for both DFS and OS on multivariate analysis (Tables 3 and 4). Moreover, although circulating ALC could predict survival outcomes, the LMR was shown to outperform ALC. In a similar study by Cummings et al., the monocyte-lymphocyte 
ratio, the reciprocal of the LMR, was not an independent prognostic factor for OS [15].

Although the precise mechanisms of the association between lower LMR and poor outcome have not been clarified, LMR is thought to reflect the balance between the favorable prognostic effect of lymphocytes and the unfavorable role of monocytes with respect to cancer progression [23]. Lymphocytes play important roles in defense against cancer cells by inducing apoptosis and suppressing proliferation and migration of cancer cells $[44,48]$. The CD3+ $\mathrm{T}$ cells and natural killer (NK) cells exhibit potent anti-cancer activities by inhibiting growth and metastasis of cancer cells [49]. Prognostic significance of peripheral lymphocyte count in various kinds of cancers has been reported $[21,50]$. In the present study, ALC was a prognostic factor for both DFS and OS on univariate analysis, although not on multivariate analysis. Monocytes are another important component of peripheral blood. Inflammation can trigger the mobilization of monocytes from the bone marrow to the peripheral blood [51]. After recruitment into tumor tissue, monocytes can differentiate into tumor-associated macrophages (TAMs) [52, 53]. Circulating monocytes in the blood may reflect the presence of TAMs [25]. In a study by Matsuo et al., elevated monocyte counts were an independent prognostic factor for DFS and OS in patients with EC [12]. In the current study, the AMC was a prognostic variable for both DFS and OS, but the significance was lost on multivariate analysis (Tables 3 and 4).

Histologic grade is one of the important factors associated with extrauterine spread and survival. Fortunately, the majority of ECs are present as low-grade tumors that tend to limit their spread to the surface of the endometrium, with a low likelihood of metastatic extension or need for adjuvant therapy [54]. Histologic grade was reported to be an independent prognostic factor for both DFS [12, 55-57] and OS $[12,55-60]$ in EC. In the present study, we also found histologic grade to be a predictor of DFS on multivariate analysis, as reported in previous studies $[12,55-57]$. However, histologic grade was not a predictor of OS in the present study, as found in previous reports [14, 61].

The strength of the current study is that it represents the first attempt to evaluate the prognostic value of the LMR in patients with EC. It is worth noting that the optimum cutoff point for LMR determined in the current study delineates a relatively small subset of patients as high risk, although this subset was associated with predominantly poor outcomes. Moreover, the value of the LMR was evaluated together with previously validated biomarkers, namely the NLR and PLR. In addition, our study was conducted at multiple institutions. Finally, by performing simple and low-cost peripheral blood examinations, it might be possible to identify patients who are at high risk of experiencing relapse or death after the standard treatment.

This study had some limitations that should be addressed, including its retrospective nature and the inclusion of a relatively small number of patients. Potential confounding biases may have negatively affected the accuracy of the results. Moreover, the median follow-up duration was rather short. In addition, the tumor types and stages included in this study were heterogeneous. Another limitation was that the LMR may be a non-specific marker of inflammation, and the results may have been affected by the presence of other systemic diseases [62]. To better understand the prognostic role of the LMR and to apply this convenient, simple, and inexpensive prognostic factor for risk stratification, additional large-scale investigations should be conducted.

In conclusion, we found that an elevated LMR was an independent prognostic factor for DFS and OS, as determined by multivariate analysis using the Cox model. Therefore, the LMR may be clinically reliable, and thus useful for the accurate prediction of prognosis in EC.

\section{Acknowledgements}

"This study was supported by a grant from the National R\&D Program for Cancer Control, Ministry for Health, Welfare, and Family Affairs, Republic of Korea (0920050)".

\section{Competing Interests}

The authors have declared that no competing interest exists.

\section{References}

1. Siegel RL, Miller KD, Jemal A. Cancer statistics, 2015. CA. 2015; 65: 5-29.

2. Jemal A, Tiwari RC, Murray T, Ghafoor A, Samuels A, Ward E, et al. Cancer statistics, 2004. CA. 2004; 54: 8-29.

3. Lewin SN, Herzog TJ, Barrena Medel NI, Deutsch I, Burke WM, Sun X, et al. Comparative performance of the 2009 international Federation of gynecology and obstetrics' staging system for uterine corpus cancer. Obstet Gynecol. 2010; 116: 1141-9.

4. Hendrickson M, Ross J, Eifel PJ, Cox RS, Martinez A, Kempson R. Adenocarcinoma of the endometrium: analysis of 256 cases with carcinoma limited to the uterine corpus. Pathology review and analysis of prognostic variables. Gynecol Oncol. 1982; 13: 373-92.

5. Nori D, Hilaris BS, Tome M, Lewis JL, Jr., Birnbaum S, Fuks Z. Combined surgery and radiation in endometrial carcinoma: an analysis of prognostic factors. Int J Radiat Oncol Biol Phys. 1987; 13: 489-97.

6. Takeshima N, Hirai Y, Tanaka N, Yamawaki T, Yamauchi K, Hasumi K. Pelvic lymph node metastasis in endometrial cancer with no myometrial invasion. Obstet Gynecol. 1996; 88: 280-2.

7. Ingram SS, Rosenman J, Heath R, Morgan TM, Moore D, Varia M. The predictive value of progesterone receptor levels in endometrial cancer. Int J Radiat Oncol Biol Phys. 1989; 17: 21-7.

8. Hanson MB, van Nagell JR, Jr., Powell DE, Donaldson ES, Gallion H, Merhige $\mathrm{M}$, et al. The prognostic significance of lymph-vascular space invasion in stage I endometrial cancer. Cancer. 1985; 55: 1753-7.

9. Morrow CP, Bundy BN, Kurman RJ, Creasman WT, Heller P, Homesley HD, et al. Relationship between surgical-pathological risk factors and outcome in 
clinical stage I and II carcinoma of the endometrium: a Gynecologic Oncology Group study. Gynecol Oncol. 1991; 40: 55-65.

10. Suh DH, Kim HS, Chung HH, Kim JW, Park NH, Song YS, et al. Pre-operative systemic inflammatory response markers in predicting lymph node metastasis in endometrioid endometrial adenocarcinoma. Eur J Obstet Gynecol Reprod Biol. 2012; 162: 206-10.

11. Ekici H, Malatyalioglu E, Kokcu A, Kurtoglu E, Tosun M, Celik H. Do Leukocyte and Platelet Counts Have Benefit for \Preoperative Evaluation of Endometrial Cancer? Asian Pac J Cancer Prev. 2015; 16: 5305-10.

12. Matsuo K, Hom MS, Moeini A, Machida H, Takeshima N, Roman LD, et al. Significance of monocyte counts on tumor characteristics and survival outcome of women with endometrial cancer. Gynecol Oncol. 2015; 138: 332-8.

13. Metindir J, Bilir Dilek G. Preoperative hemoglobin and platelet count and poor prognostic factors in patients with endometrial carcinoma. J Cancer Res Clin Oncol. 2009; 135: 125-9.

14. Heng S, Benjapibal M. Preoperative thrombocytosis and poor prognostic factors in endometrial cancer. Asian Pac J Cancer Prev. 2014; 15: 10231-6.

15. Cummings M, Merone L, Keeble C, Burland L, Grzelinski M, Sutton K, et al. Preoperative neutrophil:lymphocyte and platelet:lymphocyte ratios predict endometrial cancer survival. Br J Cancer. 2015; 113: 311-20.

16. Haruma T, Nakamura K, Nishida T, Ogawa C, Kusumoto T, Seki N, et al. Pre-treatment neutrophil to lymphocyte ratio is a predictor of prognosis in endometrial cancer. Anticancer Res. 2015; 35: 337-43.

17. Wang D, Yang JX, Cao DY, Wan XR, Feng FZ, Huang HF, et al. Preoperative neutrophil-lymphocyte and platelet-lymphocyte ratios as independent predictors of cervical stromal involvement in surgically treated endometrioid adenocarcinoma. Onco Targets Ther. 2013; 6: 211-6.

18. Li ZM, Huang JJ, Xia Y, Sun J, Huang Y, Wang Y, et al. Blood lymphocyte-to-monocyte ratio identifies high-risk patients in diffuse large B-cell lymphoma treated with R-CHOP. PloS one. 2012; 7: e41658.

19. Wang L, Wang H, Xia ZJ, Huang HQ, Jiang WQ, Lin TY, et al. Peripheral blood lymphocyte to monocyte ratio identifies high-risk adult patients with sporadic Burkitt lymphoma. Ann Hematol. 2015; 94: 1645-54.

20. Li YL, Gu KS, Pan YY, Jiao Y, Zhai ZM. Peripheral blood lymphocyte/monocyte ratio at the time of first relapse predicts outcome for patients with relapsed or primary refractory diffuse large B-cell lymphoma. BMC cancer. 2014; 14: 341

21. Lin GN, Peng JW, Liu DY, Xiao JJ, Chen YQ, Chen XQ. Increased lymphocyte to monocyte ratio is associated with better prognosis in patients with newly diagnosed metastatic nasopharyngeal carcinoma receiving chemotherapy. Tumour Biol. 2014; 35: 10849-54.

22. Jiang R, Cai XY, Yang ZH, Yan Y, Zou X, Guo L, et al. Elevated peripheral blood lymphocyte-to-monocyte ratio predicts a favorable prognosis in the patients with metastatic nasopharyngeal carcinoma. Chin J Cancer. 2015; 34: 237-46.

23. Li J, Jiang R, Liu WS, Liu Q, Xu M, Feng QS, et al. A large cohort study reveals the association of elevated peripheral blood lymphocyte-to-monocyte ratio with favorable prognosis in nasopharyngeal carcinoma. PloS one. 2013; 8: e83069.

24. Ni XJ, Zhang XL, Ou-Yang QW, Qian GW, Wang L, Chen S, et al. An elevated peripheral blood lymphocyte-to-monocyte ratio predicts favorable response and prognosis in locally advanced breast cancer following neoadjuvant chemotherapy. PloS one. 2014; 9: e111886.

25. Hu P, Shen H, Wang G, Zhang P, Liu Q, Du J. Prognostic significance of systemic inflammation-based lymphocyte- monocyte ratio in patients with lung cancer: based on a large cohort study. PloS one. 2014; 9: e108062.

26. Lin GN, Peng JW, Xiao JJ, Liu DY, Xia ZJ. Prognostic impact of circulating monocytes and lymphocyte-to-monocyte ratio on previously untreated metastatic non-small cell lung cancer patients receiving platinum-based doublet. Med Oncol. 2014; 31: 70

27. Go SI, Kim RB, Song HN, Kang MH, Lee US, Choi HJ, et al. Prognostic significance of the lymphocyte-to-monocyte ratio in patients with small cell lung cancer. Med Oncol. 2014; 31: 323.

28. Han LH, Jia YB, Song QX, Wang JB, Wang NN, Cheng YF. Prognostic significance of preoperative lymphocyte-monocyte ratio in patients with resectable esophageal squamous cell carcinoma. Asian Pac J Cancer Prev. 2015; 16: 2245-50.

29. Huang Y, Feng JF. Low preoperative lymphocyte to monocyte ratio predicts poor cancer-specific survival in patients with esophageal squamous cell carcinoma. Onco Targets Ther. 2015; 8: 137-45.

30. Zhou X, Du Y, Xu J, Huang Z, Qiu T, Wang X, et al. The preoperative lymphocyte to monocyte ratio predicts clinical outcomes in patients with stage II/III gastric cancer. Tumour Biol. 2014; 35: 11659-66.

31. Deng $Q$, He B, Liu X, Yue J, Ying H, Pan Y, et al. Prognostic value of pre-operative inflammatory response biomarkers in gastric cancer patients and the construction of a predictive model. J Transl Med. 2015; 13: 66.

32. Ozawa T, Ishihara S, Kawai K, Kazama S, Yamaguchi H, Sunami E, et al. Impact of a lymphocyte to monocyte ratio in stage IV colorectal cancer. J Surg Res. 2015; 199: 386-92.

33. Stotz M, Pichler M, Absenger G, Szkandera J, Arminger F, Schaberl-Moser R, et al. The preoperative lymphocyte to monocyte ratio predicts clinical outcome in patients with stage III colon cancer. Br J Cancer. 2014; 110: 435-40.

34. Qi Q, Geng Y, Sun M, Wang P, Chen Z. Clinical implications of systemic inflammatory response markers as independent prognostic factors for advanced pancreatic cancer. Pancreatology. 2015; 15: 145-50.
35. Stotz M, Szkandera J, Stojakovic T, Seidel J, Samonigg H, Kornprat P, et al. The lymphocyte to monocyte ratio in peripheral blood represents a novel prognostic marker in patients with pancreatic cancer. Clin Chem Lab Med. 2015; 53: 499-506.

36. Zhang GM, Zhu Y, Luo L, Wan FN, Zhu YP, Sun LJ, et al. Preoperative lymphocyte-monocyte and platelet-lymphocyte ratios as predictors of overall survival in patients with bladder cancer undergoing radical cystectomy. Tumour Biol. 2015; 36: 8537-43.

37. Chen L, Zhang F, Sheng XG, Zhang SQ. Decreased pretreatment lymphocyte/monocyte ratio is associated with poor prognosis in stage Ib1-Ila cervical cancer patients who undergo radical surgery. Onco Targets Ther. 2015; 8: 1355-62

38. Pecorelli S. Revised FIGO staging for carcinoma of the vulva, cervix, and endometrium. Int J Gynaecol Obstet. 2009; 105: 103-4.

39. Williams KA, Labidi-Galy SI, Terry KL, Vitonis AF, Welch WR, Goodman A, et al. Prognostic significance and predictors of the neutrophil-to-lymphocyte ratio in ovarian cancer. Gynecol Oncol. 2014; 132: 542-50.

40. Wright JD, Barrena Medel NI, Sehouli J, Fujiwara K, Herzog TJ. Contemporary management of endometrial cancer. Lancet. 2012; 379: 1352-60.

41. Raungkaewmanee S, Tangjitgamol S, Manusirivithaya S, Srijaipracharoen S, Thavaramara T. Platelet to lymphocyte ratio as a prognostic factor for epithelial ovarian cancer. J Gynecol Oncol. 2012; 23: 265-73.

42. Takahashi R, Mabuchi S, Kawano M, Sasano T, Matsumoto Y, Kuroda H, et al. Prognostic significance of systemic neutrophil and leukocyte alterations in surgically treated endometrial cancer patients: a monoinstitutional study. Gynecol Oncol. 2015; 137: 112-8.

43. Balkwill F, Mantovani A. Inflammation and cancer: back to Virchow? Lancet. 2001; 357: 539-45.

44. Coussens LM, Werb Z. Inflammation and cancer. Nature. 2002; 420: 860-7.

45. Grivennikov SI, Greten FR, Karin M. Immunity, inflammation, and cancer. Cell. 2010; 140: 883-99.

46. Kim SI, Kim HS, Kim TH, Suh DH, Kim K, No JH, et al. Impact of underweight after treatment on prognosis of advanced-stage ovarian cancer. J Immunol Res. 2014; 2014: 349546

47. Li J, Lin J, Luo Y, Kuang M, Liu Y. Multivariate Analysis of Prognostic Biomarkers in Surgically Treated Endometrial Cancer. PloS one. 2015; 10: e0130640.

48. Mantovani A, Allavena P, Sica A, Balkwill F. Cancer-related inflammation. Nature. 2008; 454: 436-44.

49. Ohashi R, Takahashi K, Miura K, Ishiwata T, Sakuraba S, Fukuchi Y. Prognostic factors in patients with inoperable non-small cell lung cancer--an analysis of long-term survival patients. Gan To Kagaku Ryoho. 2006; 33: 1595-602.

50. Huang JJ, Li YJ, Xia Y, Wang Y, Wei WX, Zhu YJ, et al. Prognostic significance of peripheral monocyte count in patients with extranodal natural killer/T-cell lymphoma. BMC Cancer. 2013; 13: 222.

51. Shi C, Pamer EG. Monocyte recruitment during infection and inflammation. Nat Rev Immunol. 2011; 11: 762-74.

52. Pollard JW. Tumour-educated macrophages promote tumour progression and metastasis. Nat Rev Cancer. 2004; 4: 71-8.

53. Hagemann $\mathrm{T}$, Lawrence $\mathrm{T}$. Investigating macrophage and malignant cell interactions in vitro. Methods Mol Biol. 2009; 512: 325-32.

54. Chan JK, Kapp DS, Cheung MK, Shin JY, Stieglitz D, Husain A, et al. Prognostic factors and risk of extrauterine metastases in 3867 women with grade 1 endometrioid corpus cancer. Am J Obstet Gynecol. 2008; 198: 216.e1-5.

55. Liu YD, Dai M, Yang SS, Xiao M, Meng FL, Chen XW. Overexpression of Lysine-Specific Demethylase 1 Is Associated With Tumor Progression and Unfavorable Prognosis in Chinese Patients With Endometrioid Endometrial Adenocarcinoma. Int J Gynecol Cancer. 2015; 25: 1453-60.

56. Liu Y, Meng F, Xu Y, Yang S, Xiao M, Chen X, et al. Overexpression of Wnt7a is associated with tumor progression and unfavorable prognosis in endometrial cancer. Int J Gynecol Cancer. 2013; 23: 304-11.

57. Guntupalli SR, Zighelboim I, Kizer NT, Zhang Q, Powell MA, Thaker PH, et al. Lymphovascular space invasion is an independent risk factor for nodal disease and poor outcomes in endometrioid endometrial cancer. Gynecol Oncol. 2012; 124: 31-5.

58. Huang CY, Ho CM, Chen YL, You SL, Chen CA, Cheng WF. Impact of lymphadenectomy in uterine endometrioid carcinoma. Eur J Surg Oncol. 2013; 39: 350-7.

59. Zighelboim I, Ali S, Lankes HA, Backes F, Moore K, Mutch D, et al. Assessing the prognostic role of ATR mutation in endometrioid endometrial cancer: An NRG Oncology/Gynecologic Oncology Group study. Gynecol Oncol. 2015; 138: 614-9.

60. Meng FL, Yin MZ, Song HT, Yang H, Lou G, Zhou RL. LAPTM4B-35 overexpression is an independent prognostic marker in endometrial carcinoma. Int $\mathrm{J}$ Gynecol Cancer. 2010; 20: 745-50.

61. Gorelick C, Andikyan V, Mack M, Lee YC, Abulafia O. Prognostic significance of preoperative thrombocytosis in patients with endometrial carcinoma in an inner-city population. Int J Gynecol Cancer. 2009; 19: 1384-9.

62. Madjid M, Fatemi O. Components of the complete blood count as risk predictors for coronary heart disease: in-depth review and update. Tex Heart Inst J. 2013; 40: 17-29. 